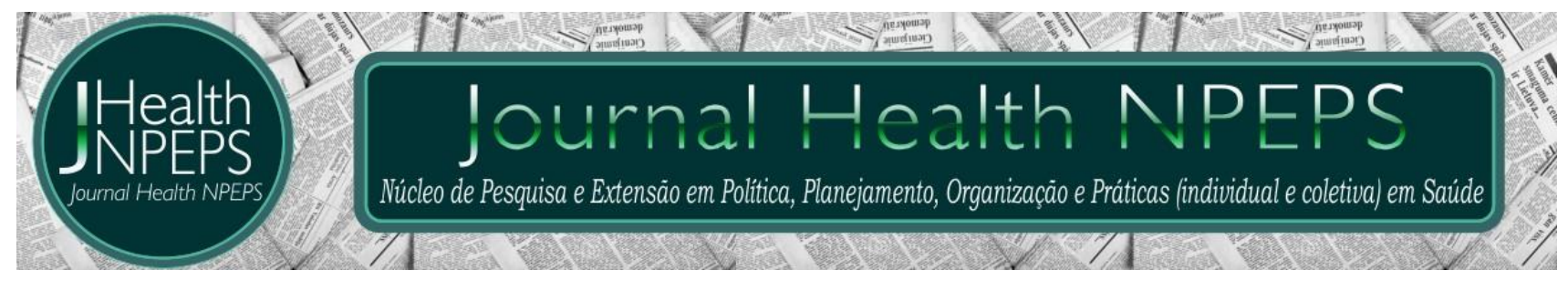

http://dx.doi.org/10.30681/252610105002

ARTIGO ORIGINAL

\title{
Avaliação da atenção básica: estudo ecológico dos indicadores de pactuação em Santa Catarina
}

\section{Evaluation of basic care: ecological study of pactuation indicators in Santa Catarina
Evaluación de cuidados básicos: estudio ecológico de los indicadores de pactuación en Santa Catarina

\section{Sabrina da Silva de Souza ${ }^{1}$, Pedro Fredemir Palha², Ana Cláudia Cunha ${ }^{3}$, Dione Lúcia Prim Laurindo ${ }^{4}$, Samara Eliane Rabelo Suplici ${ }^{5}$, katheri Maris Zamprogna ${ }^{6}$}

\section{RESUMO}

Objetivo: avaliar a cobertura de atenção básica nos municípios de Santa Catarina e o alcance dos indicadores de pactuação. Método: estudo ecológico transversal, usando a técnica de análise espacial, realizada no período de 2017 e 2018, no estado de Santa Catarina. Para a análise, utilizou-se média, mediana e desvio padrão para todos os indicadores do estudo. Resultados: o estudo apresentou três blocos de observação, o primeiro ocupou em analisar os indicadores diretamente correlacionados à cobertura de atenção básica, o segundo, aos indicadores inversamente correlacionados e o por último, aqueles que não foram influenciados. Conclusão: apesar da cobertura populacional estimada pelas equipes de atenção básica não influenciar todos os indicadores interfederativos, há uma relação positiva se comparado à melhoria de alguns deles. O desempenho dos municípios com melhor cobertura populacional estimada pelas equipes de atenção básica nem sempre apresentam os melhores indicadores de saúde.

Descritores: Avaliação em Saúde; Indicadores Básicos de Saúde; Enfermagem.

\section{ABSTRACT}

${ }^{1}$ Enfermeira. Doutora em enfermagem. Universidade Federal de Santa Catarina. São José, Santa Catarina, Brasil. E-mail: enfermeirasabrina@gmail.com ORCID ID: https://orcid.org/0000-0001-9046-6434 Autor para correspondência - Endereço: Rua Elizei di Bernardi 200 apto 406 A. São José, Santa Catarina, Brasil.

${ }^{2}$ Enfermeiro. Doutor em enfermagem. Universidade de São Paulo (USP). Ribeirão Preto, São Paulo, Brasil. E-mail: pedropalha@gmail.com ORCID ID: https://orcid.org/0000-0002-5220-4529

3 Enfermeira. Especialista. Secretaria Municipal de São José. São José, Santa Catarina, Brasil. E-mail: cau cunha@yahoo.com ORCID ID: http://orcid.org/0000-0003-2292-3917

${ }^{4}$ Enfermeira. Especialista. Secretaria Municipal de São José. São José, Santa Catarina, Brasil. E-mail: dione-prim@gmail.com ORCID ID: http://orcid.org/0000-0001-6233-2527

${ }^{5}$ Enfermeira. Doutora em Enfermagem. Florianópolis, Santa Catarina, Brasil. E-mail: samara.suplici@gmail.com ORCID ID: http://orcid.org/0000-0002-0334-7195

${ }^{6}$ Enfermeira. Doutora em Enfermagem. Secretaria Municipal de São José. São José, Santa Catarina, Brasil. E-mail: katherizamprogna@gmail.com ORCID ID: http://orcid.org/0000-0002-5987-1025

Este artigo está licenciado sob forma de uma licença Creative Commons Atribuição 4.0 Internacional, que permite uso irrestrito, distribuição e reprodução em qualquer meio, desde que a publicação original seja corretamente citada. 
Objective: to evaluate the coverage of primary care in the municipalities of Santa Catarina and the reach of the agreement indicators. Method: cross-sectional ecological study, using the spatial analysis technique, carried out between 2017 and 2018, in the state of Santa Catarina. For the analysis, mean, median and standard deviation were used for all the indicators of the study. Results: the study presented three blocks of observation, the first concerned analyzing the indicators directly correlated to primary care coverage, the second, the inversely correlated indicators and the last, those that were not influenced. Conclusion: although the population coverage estimated by the primary care teams does not influence all the inter-federative indicators, there is a positive relationship when compared to the improvement of some of them. The performance of the municipalities with the best population coverage estimated by the primary care teams does not always present the best health indicators.

Descriptors: Health Evaluation; Basic Health Indicators; Nursing.

\section{RESUMEN}

Objetivo: evaluar la cobertura de la atención primaria en los municipios de Santa Catarina y el alcance de los indicadores de acuerdo. Método: estudio ecológico transversal, mediante la técnica de análisis espacial, realizado entre 2017 y 2018, en el estado de Santa Catarina. Para el análisis, usamos media, mediana, desviación estándar para todos los indicadores del estudio. Resultados: el estudio presentó tres bloques de observación, el primero se preocupó por analizar los indicadores directamente correlacionados con la cobertura de atención primaria, el segundo, los indicadores inversamente correlacionados y el último, los que no fueron influenciados. Conclusión: si bien la cobertura poblacional estimada por los equipos de atención primaria no influye en todos los indicadores inter-federativos, se observa que existe una relación positiva al compararlos con la mejora de algunos de ellos. El desempeño de los municipios con mejor cobertura poblacional estimada por los equipos de atención primaria no siempre presenta los mejores indicadores de salud.

Descriptores: Evaluación en Salud; Indicadores de Salud; Enfermería.

\section{INTRODUÇÃO}

As demandas e as necessidades de atenção à saúde advindas das mudanças no perfil demográfico e epidemiológico da população se relacionam diretamente ao acesso, à integralidade, à efetividade e aos custos das ações de saúde ${ }^{1}$. A oportunidade e pertinência dos princípios filosóficos e operacionais do Sistema Único de Saúde (SUS) implicam na organização de serviços de saúde, descentralizados e articulados por nível de complexidade 2 .

$$
\text { É nessa lógica que os sistemas }
$$
de saúde públicos têm fomentado ações voltadas à atenção primária ${ }^{3}$. Isso se justifica, pelas características deste nível de atenção que aproxima a equipe de saúde do usuário, com possibilidade de ampliar a adesão aos tratamentos e orientações, e diminuir as intervenções em níveis que geram custos mais 
elevados, como a média e alta complexidade ${ }^{3}$.

A pactuação de responsabilidades e metas é parte indissociável do processo do planejamento em saúde. Anualmente, municípios, estados e governo federal definem e pactuam metas para um conjunto de indicadores, que representam as responsabilidades dos gestores, buscando viabilizar o seu cumprimento e a qualificação de áreas estratégicas para a saúde 4 .

Os indicadores de saúde são medidas sintéticas que contêm informações relevantes e refletem o quadro real das condições e das dimensões de saúde da população, bem como do desempenho do sistema de saúde ${ }^{5}$. A Resolução CIT no 8 de 2016 dispõe sobre o processo de pactuação interfederativa de indicadores para os anos de 2017-20216, com um rol de 23 indicadores. Desses indicadores, 20 são de pactuação universal, ou seja, de pactuação comum e obrigatória aos municípios e estados, e três indicadores específicos ${ }^{6}$.

Para discutir os indicadores de saúde, é preciso considerar o próprio significado de saúde. Devido à sua natureza multidimensional, o conceito deve ser examinado à luz de vários referentes culturais e teóricos. Para a saúde, os indicadores são medidas sumárias que podem, de forma simples, revelar (ou medir) uma situação que não é óbvia quando considerada isoladamente, mas, é uma forma de medir as características de saúde especificadas em uma determinada população e tentam descrever e monitorar o estado de saúde de uma população ${ }^{7}$.

Promover a demanda por dados e o uso rotineiro de dados para a tomada de decisões é um passo importante para a criação de uma cultura de qualidade nos sistemas de saúde. Assim, há a necessidade de construir e utilizar indicadores de monitoramento e avaliação, com o desafio de realizar ações de saúde mais efetivas para atender aos usuários.

Tendo a avaliação como ferramenta integrante das iniciativas voltadas para a efetivação dos diversos caminhos do SUS, atribuída como estrutura decisória ${ }^{8}$, este estudo tem como hipótese: municípios com maior cobertura de atenção básica têm melhores resultados nos indicadores de pactuação interfederativa 2017-2021. Assim, teve como objetivo avaliar a 
cobertura de atenção básica nos municípios de Santa Catarina e o alcance dos indicadores de pactuação.

\section{MÉTODO}

Estudo do tipo ecológico transversal, realizado no período de 2017 e 2018, usando a técnica de análise espacial, em relação aos 295 municípios do Estado de Santa Catarina. Utilizou-se o método de registros de relacionamento probabilístico, visando a analisar a cobertura de saúde da atenção básica e os 21 indicadores da pactuação interfederativa nos anos de 2017 e 2018.

Os dados foram extraídos do bancos de dados oficiais do Estado de Santa Catarina, disponíveis e de acesso público da DIVE/SC, em 23 de novembro de 20199. Como variável independente foi utilizada a cobertura populacional estimada pelas equipes de atenção básica. Os demais 21 indicadores foram tratados como variáveis dependentes.

0 processo de análise de dados da presente pesquisa iniciou com exploração descritiva, incluindo média, mediana, desvio padrão para todos os indicadores de estudo. Para testar a hipótese de que os indicadores selecionados não eram correlacionados entre si foi proposta uma matriz de correlação a partir do cálculo do coeficiente de correlação de Spearman. Foram plotados ainda mapas coropléticos com indicadores selecionados para avaliação a distribuição dos indicadores na área de estudo no período de 2017 e 2018. Os mapas foram desenvolvidos no software Quantum Geographic Information Sysstem (QGIS), uma multiplataforma de sistema de informação geográfica, de acesso livre, que permite a visualização, edição e análise de dados georreferenciados ${ }^{10}$. Todos os testes foram realizados com apoio computacional dos softwares R, IBM SPSS 25 (Statistical Package for the Social Sciences) e Excel 2016 ® (Microsoft Office) levando em consideração um a bidirecional de 0.05 e um intervalo de confiança (IC) de $95 \%$ e. Os mapas foram desenvolvidos no software QGIS3.10.2.

A pesquisa respeitou todos os aspectos éticos, de acordo com a resolução 466/12 do Conselho Nacional de Saúde (CNS), para esse tipo de investigação baseada em dados secundários. 


\section{RESULTADOS}

$\mathrm{Na}$ Tabela 1, observa-se que seis indicadores mantiveram a mesma média nos anos de 2017 e 2018, tendo sido registrado uma média de 69 óbitos por doença crônica não transmissível no Estado de Santa Catarina, média de quatro casos de sífilis congênita, de zero casos de aids em menores de um ano, de zero óbitos maternos, razão média de seis exames de colo de útero e razão média de zero exames de mamografia. Foram registrados em 2017, 612 casos novos de sífilis congênita, três casos de aids em menores de cinco anos de idade e 29 óbitos maternos. No ano de 2018, 623 casos novos de sífilis congênita, cinco casos de aids em menores de cinco anos de idade e 27 óbitos maternos. Aumento na taxa de mortalidade prematura por doença crônica não transmissível em 2018 (312,7).

No ano de 2018, os indicadores percentual de óbitos de mulheres em idade fértil investigados, percentual de óbitos por causa básica definida, percentual de vacina com cobertura adequada, proporção de casos de doenças de notificação encerradas, percentual de cura da hanseníase, cobertura populacional estimada de equipes de saúde da família, cobertura populacional estimada de equipes de equipe de atenção básica, realização das ações de vigilância sanitária (VISA), apresentaram médias menores que no ano de 2017.

Os indicadores com médias melhores em 2018 do que em 2017 foram: percentual de gravidez na adolescência, percentual de parto normal, número de óbitos infantis, cobertura do programa bolsa família, ações de matriciamento do CAPS, proporção de preenchimento "ocupação" nas notificações de agravos relacionados ao trabalho.

$\mathrm{Na}$ Figura 1, o número de óbitos por doença crônica não transmissível (V4) está inversamente correlacionado à cobertura populacional estimada pelas equipes de atenção básica (V23), tanto para o ano de 2017 quanto para o ano de 2018. 
Tabela 1 - Tabela descritiva dos diferentes indicadores (2017-2018).

\begin{tabular}{|c|c|c|c|c|c|c|c|c|c|c|c|c|c|c|}
\hline & \multicolumn{7}{|c|}{2017} & \multicolumn{7}{|c|}{2018} \\
\hline & Média & DP & Mediana & $\mathrm{P} 25$ & $\mathrm{P} 75$ & Mínimo & Máximo & Média & DP & Mediana & $\mathrm{P} 25$ & P75 & Mínimo & Máximo \\
\hline $\begin{array}{l}\text { Número de óbitos por doença } \\
\text { crônica não transmissível }\end{array}$ & 69 & 595 & 12 & 6 & 28 & 0 & 10195 & 69 & 602 & 12 & 6 & 28 & 0 & 10306 \\
\hline $\begin{array}{l}\text { Taxa de mortalidade prematura } \\
\text { por doença crônica não } \\
\text { transmissível }\end{array}$ & 312,0 & 127,9 & 305,5 & 234,9 & 387,5 & 0,0 & 713,8 & 312,7 & 126,7 & 304,3 & 231,2 & 373,3 & 0,0 & 789,7 \\
\hline $\begin{array}{l}\% \text { de óbitos em mulheres em } \\
\text { idade fértil investigados }\end{array}$ & $76,0 \%$ & $41,5 \%$ & $100,0 \%$ & $71,4 \%$ & $100,0 \%$ & $0,0 \%$ & $100,0 \%$ & $70,5 \%$ & $43,9 \%$ & $100,0 \%$ & $0,0 \%$ & $100,0 \%$ & $0,0 \%$ & $100,0 \%$ \\
\hline $\begin{array}{l}\% \text { de óbitos por causa básica } \\
\text { definida }\end{array}$ & $96,1 \%$ & $5,0 \%$ & $97,5 \%$ & $94,4 \%$ & $100,0 \%$ & $50,0 \%$ & $100,0 \%$ & $95,1 \%$ & $5,0 \%$ & $96,4 \%$ & $92,9 \%$ & $98,8 \%$ & $74,1 \%$ & $100,0 \%$ \\
\hline $\begin{array}{l}\% \text { de vacina com cobertura } \\
\text { adequada }\end{array}$ & $60,6 \%$ & $40,7 \%$ & $75,0 \%$ & $25,0 \%$ & $100,0 \%$ & $0,0 \%$ & $100,0 \%$ & $55,7 \%$ & $40,1 \%$ & $50,0 \%$ & $25,0 \%$ & $100,0 \%$ & $0,0 \%$ & $100,0 \%$ \\
\hline $\begin{array}{l}\text { Proporção de casos de } \\
\text { doenças de notificação } \\
\text { encerradas }\end{array}$ & $44,3 \%$ & $46,4 \%$ & $0,0 \%$ & $0,0 \%$ & $100,0 \%$ & $0,0 \%$ & $100,0 \%$ & $40,9 \%$ & $45,2 \%$ & $0,0 \%$ & $0,0 \%$ & $100,0 \%$ & $0,0 \%$ & $100,0 \%$ \\
\hline$\%$ de cura de hanseníase & $19,4 \%$ & $39,1 \%$ & $0,0 \%$ & $0,0 \%$ & $0,0 \%$ & $0,0 \%$ & $100,0 \%$ & $18,3 \%$ & $37,9 \%$ & $0,0 \%$ & $0,0 \%$ & $0,0 \%$ & $0,0 \%$ & $100,0 \%$ \\
\hline $\begin{array}{l}\text { Casos novos de sífilis } \\
\text { congênita }\end{array}$ & 4 & 36 & 0 & 0 & 1 & 0 & 612 & 4 & 37 & 0 & 0 & 1 & 0 & 623 \\
\hline $\begin{array}{l}\text { Casos de Aids em menores de } \\
5 \text { anos }\end{array}$ & 0 & 0 & 0 & 0 & 0 & 0 & 3 & 0 & 0 & 0 & 0 & 0 & 0 & 5 \\
\hline$\%$ de análise de água tratada & $93,3 \%$ & $41,2 \%$ & $91,6 \%$ & $72,8 \%$ & $108,1 \%$ & $0,0 \%$ & $250,0 \%$ & $98,8 \%$ & $45,3 \%$ & $100,0 \%$ & $66,7 \%$ & $100,0 \%$ & $0,0 \%$ & $266,7 \%$ \\
\hline $\begin{array}{l}\text { Razão de exame de colo CA } \\
\text { útero }\end{array}$ & 0,6 & 0,3 & 0,6 & 0,5 & 0,8 & 0,0 & 1,8 & 0,6 & 0,3 & 0,6 & 0,4 & 0,8 & 0,0 & 2,5 \\
\hline Razão de mamografias & 0 & 0 & 0 & 0 & 1 & 0 & 2 & 0 & 0 & 0 & 0 & 1 & 0 & 2 \\
\hline$\%$ de parto normal & $38,2 \%$ & $13,3 \%$ & $38,2 \%$ & $30,1 \%$ & $47,2 \%$ & $3,6 \%$ & $100,0 \%$ & $38,6 \%$ & $13,1 \%$ & $39,8 \%$ & $30,5 \%$ & $47,7 \%$ & $0,0 \%$ & $80,8 \%$ \\
\hline $\begin{array}{l}\% \text { de gravidez na } \\
\text { adolescência }\end{array}$ & $14,2 \%$ & $7,9 \%$ & $13,0 \%$ & $9,7 \%$ & $17,1 \%$ & $0,0 \%$ & $100,0 \%$ & $12,5 \%$ & $6,1 \%$ & $11,5 \%$ & $8,4 \%$ & $15,4 \%$ & $0,0 \%$ & $34,2 \%$ \\
\hline Número de óbitos infantis & 7 & 57 & 1 & 0 & 3 & 0 & 976 & 6 & 55 & 1 & 0 & 3 & 0 & 944 \\
\hline Taxa de Mortalidade Infantil & 11,4 & 14,5 & 8,7 & 0,0 & 15,2 & 0,0 & 97,6 & 11,6 & 14,4 & 8,1 & 0,0 & 16,5 & 0,0 & 103,5 \\
\hline Número de óbitos maternos & 0 & 2 & 0 & 0 & 0 & 0 & 29 & 0 & 2 & 0 & 0 & 0 & 0 & 27 \\
\hline $\begin{array}{l}\text { Cobertura populacional de } \\
\text { Saúde da Família }\end{array}$ & 95,8 & 12,4 & 100,0 & 100,0 & 100,0 & 0,0 & 100,0 & 95,7 & 12,8 & 100,0 & 100,0 & 100,0 & 0,0 & 100,0 \\
\hline $\begin{array}{l}\text { Cobertura do programa Bolsa } \\
\text { Família }\end{array}$ & 83,4 & 13,6 & 86,5 & 77,9 & 92,6 & 0,0 & 100,0 & 85,6 & 12,0 & 88,2 & 79,8 & 94,0 & 0,0 & 100,0 \\
\hline $\begin{array}{l}\text { Cobertura populacional de } \\
\text { equipes de Atenção Básica }\end{array}$ & 82,8 & 25,3 & 100,0 & 65,4 & 100,0 & 0,0 & 100,0 & 82,4 & 25,5 & 100,0 & 66,7 & 100,0 & 0,0 & 100,0 \\
\hline Realização de ações de VISA & 92,1 & 21,0 & 100,0 & 100,0 & 100,0 & 0,0 & 100,0 & 87,4 & 23,0 & 100,0 & 83,3 & 100,0 & 0,0 & 100,0 \\
\hline $\begin{array}{l}\text { Ações de Matriciamento do } \\
\text { CAPS }\end{array}$ & 3,3 & 17,3 & 0,0 & 0,0 & 0,0 & 0,0 & 100,0 & 4,7 & 19,9 & 0,0 & 0,0 & 0,0 & 0,0 & 100,0 \\
\hline $\begin{array}{l}\text { Proporção de preenchimento } \\
\text { "ocupação" nas notificações } \\
\text { de ART }\end{array}$ & $27,4 \%$ & $38,9 \%$ & $0,0 \%$ & $0,0 \%$ & $63,6 \%$ & $0,0 \%$ & $100,0 \%$ & $82,9 \%$ & $28,8 \%$ & $96,9 \%$ & $78,6 \%$ & $100,0 \%$ & $0,0 \%$ & $100,0 \%$ \\
\hline
\end{tabular}

DP: Desvio Padrão, P25: Percentil 25, P75: Percentil 75.

Os indicadores, taxa de mortalidade prematura por doença crônica não transmissível (V5), percentual de óbitos por causa básicas definida (V7), proporção de parto normal no SUS (V16), percentual de gravidez na adolescência (V17), taxa de mortalidade infantil (V19) e realização de ações da vigilância sanitária (V24) não foram influenciados pela cobertura populacional de atenção básica em Santa Catarina, uma vez que não apresentaram significância estatística. 

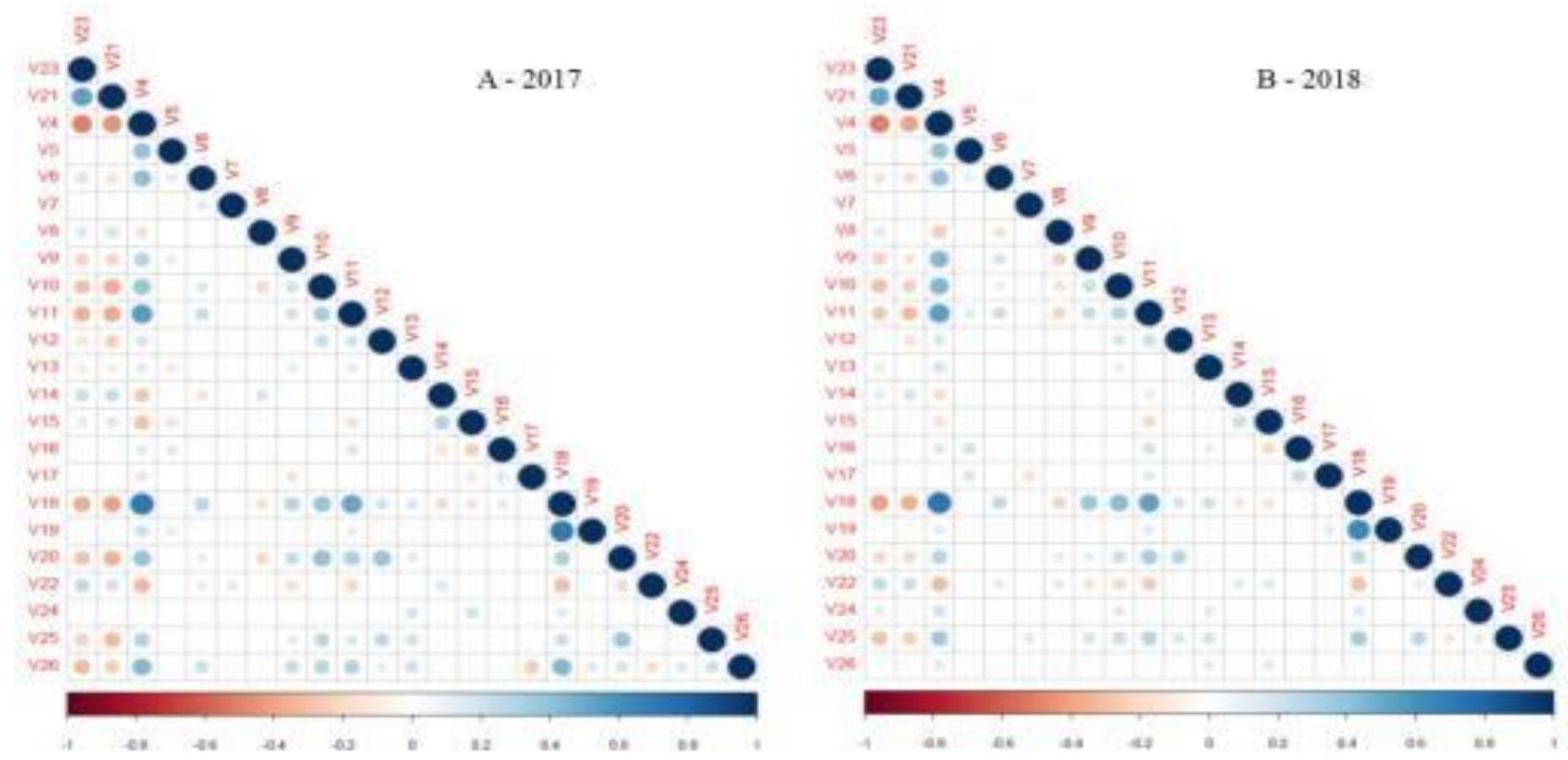

Figura 1 - Matriz de correlação dos indicadores (2017-2018).

Nota: os pontos que não apareceram na matriz foram aqueles não significantes estatisticamente $(p \geq 0,05)$. O coeficiente de correlação está na cor, quanto mais forte for a cor, mais bem correlacionado estão os indicadores. Dessa forma pode-se verificar, por exemplo, que número de óbitos por doença crônica não transmissível (V4) está inversamente correlacionado à Cobertura populacional de equipes de Atenção Básica (V23), tanto para o ano de 2017 quanto para o ano de 2018. Isso significa dizer que quanto maior foi à presença de uma menor é a presença da outra.

Os indicadores cobertura populacional de saúde da família (V21), percentual de vacina com cobertura adequada (V8), razão de exames citopatológicos de mulheres na faixa etária de 29-59 anos (V14), razão de exames de mamografia em mulheres na faixa etária de 49-69 anos (V15) e cobertura do programa bolsa família (V22) estão diretamente correlacionados à cobertura populacional estimada pelas equipes de Atenção Básica (V23), tanto para o ano de 2017 quanto para o ano de 2018.

Já os indicadores número de óbitos por doença crônica não transmissível (V4), percentual de óbitos de mulheres em idade fértil investigados (V6), proporção de casos de doenças de notificação encerrados (V9), percentual de cura de hanseníase (V10), casos novos de sífilis em menores de um ano de idade (V11), casos de aids em menores de um ano de idade (V12), percentual de análise de água realizada (V13), número de óbito infantil (V18), número de óbitos maternos (V20), ações de matriciamento do CAPS (V25) e proporção de preenchimento do campo "ocupação" nas notificações de agravos relacionados ao trabalho (V26) estão inversamente correlacionado à cobertura populacional 
estimada pelas equipes de Atenção quanto para o ano de 2018.

Básica (V23), tanto para o ano de 2017

Figura 2 - Mapas temáticos dos indicadores diretamente correlacionados à cobertura de atenção básica (2017-2018).

2017
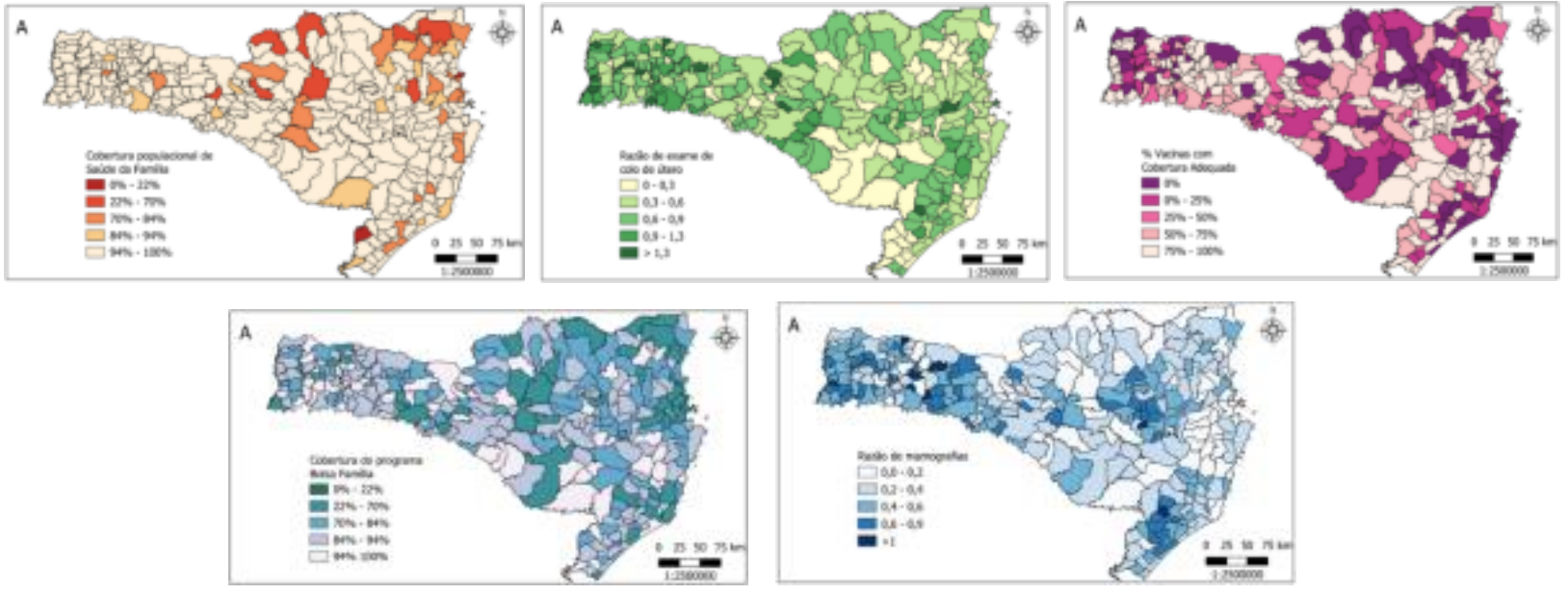

2018
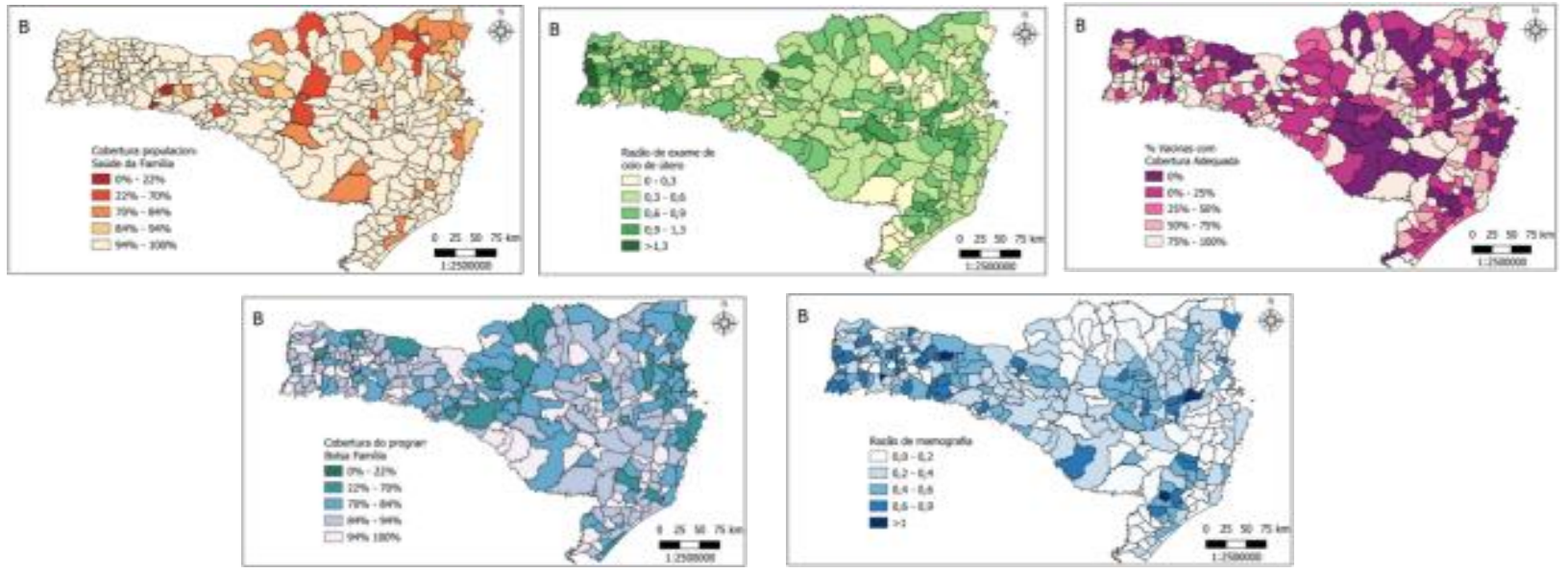

Nota: Os mapas coropléticos representam a proporcionalidade da variável estatística estudada (cada indicador individualmente). Cada unidade (município) está destacada de forma a representar a magnitude de da variável estudada, ou seja, proporcionalmente ao nível de medida da variável estatística que está sendo retratada no mapa. Os mapas coropléticos foram elaborados com dados quantitativos e regras próprias de utilização da variável visual de cor, variando sua intensidade conforme a sequência de valores apresentados nas classes estabelecidas.

Fonte: Pesquisadores, 2020. 
Figura 3 - Mapas temáticos dos indicadores inversamente correlacionados à cobertura de atenção básica (2017-2018).

2017
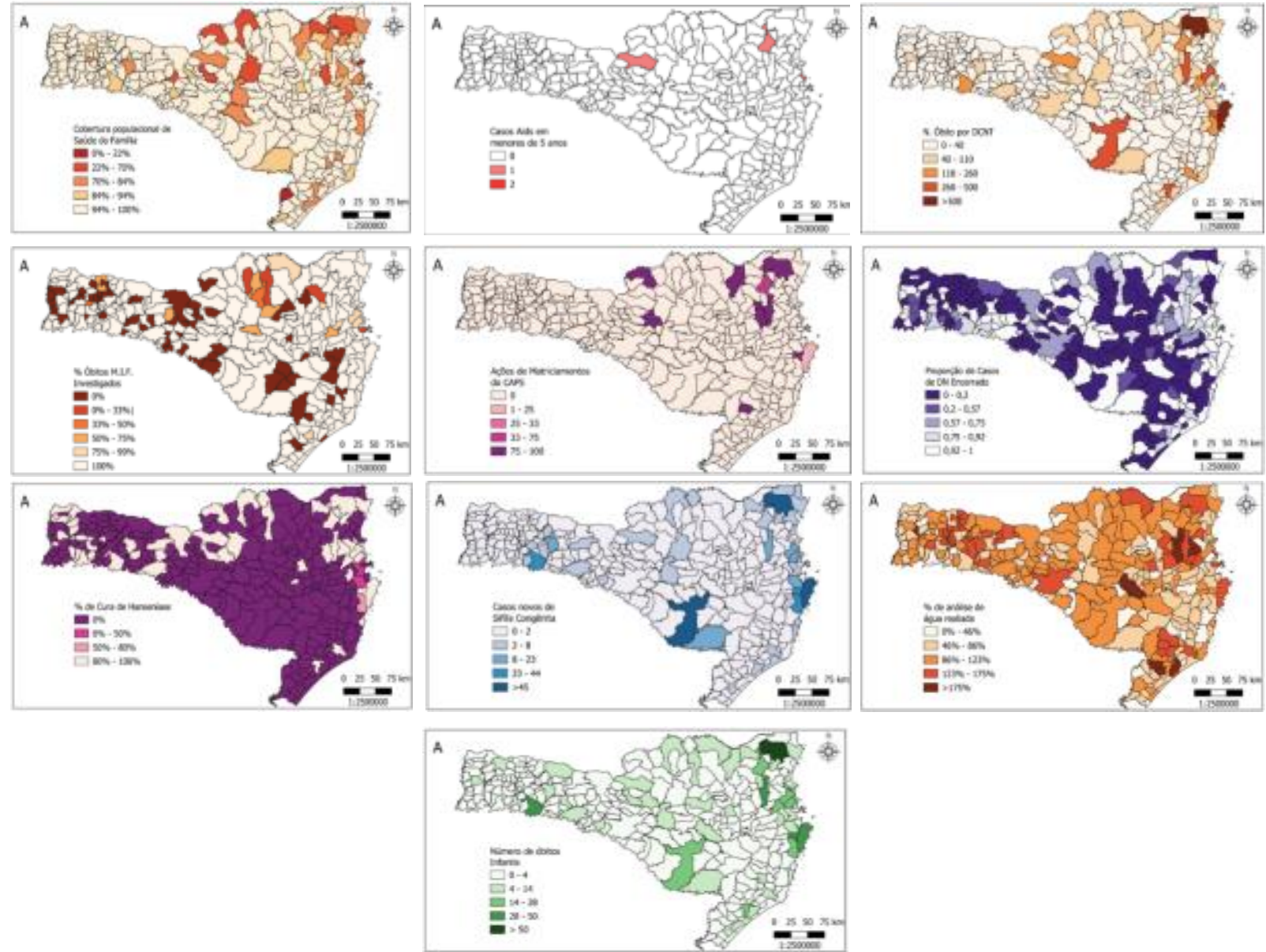

2018
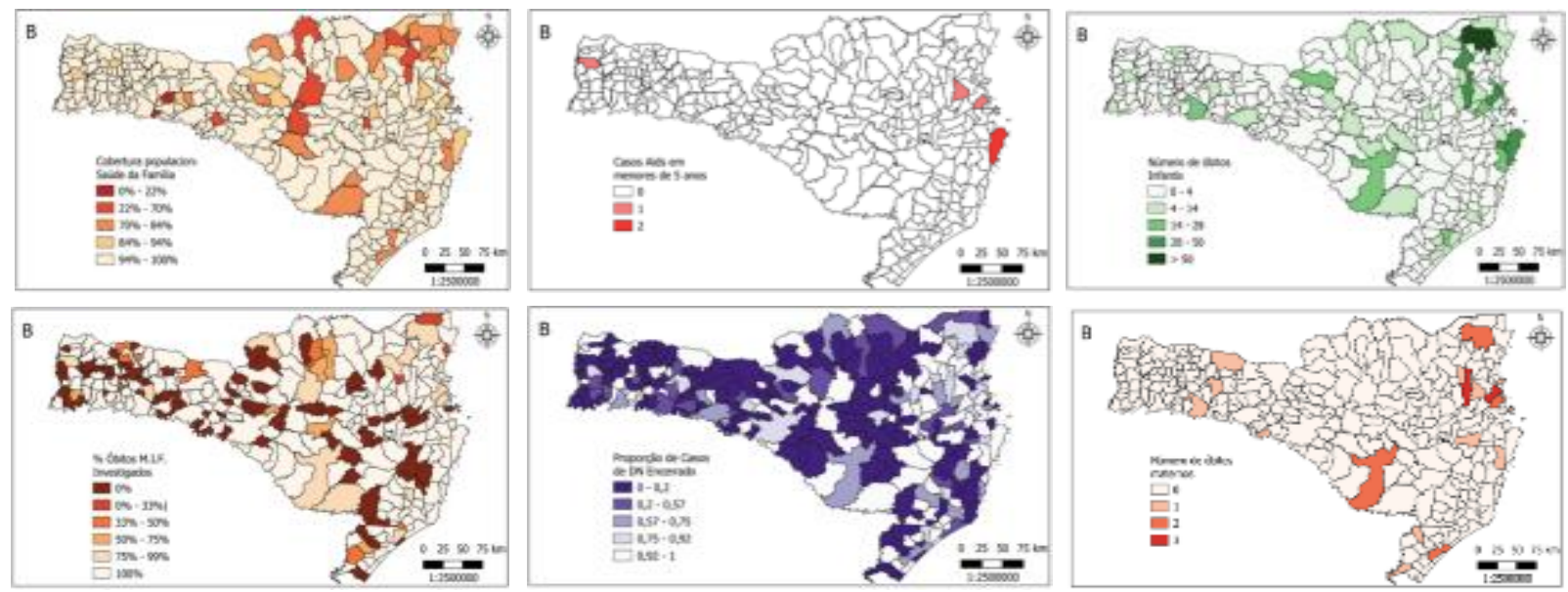

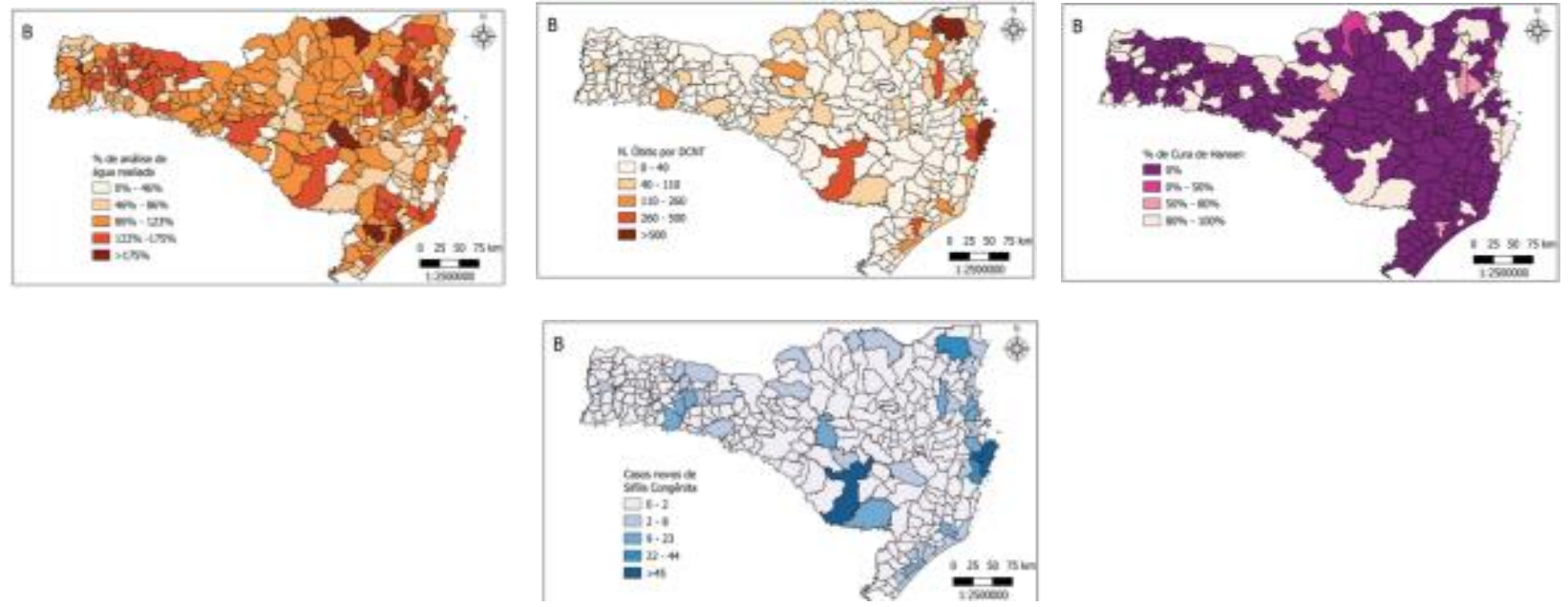

Fonte: Pesquisadores, 2020.

Figura 4 - Mapas temáticos dos indicadores que não são influenciados pela cobertura de atenção básica (2017-2018).

2017
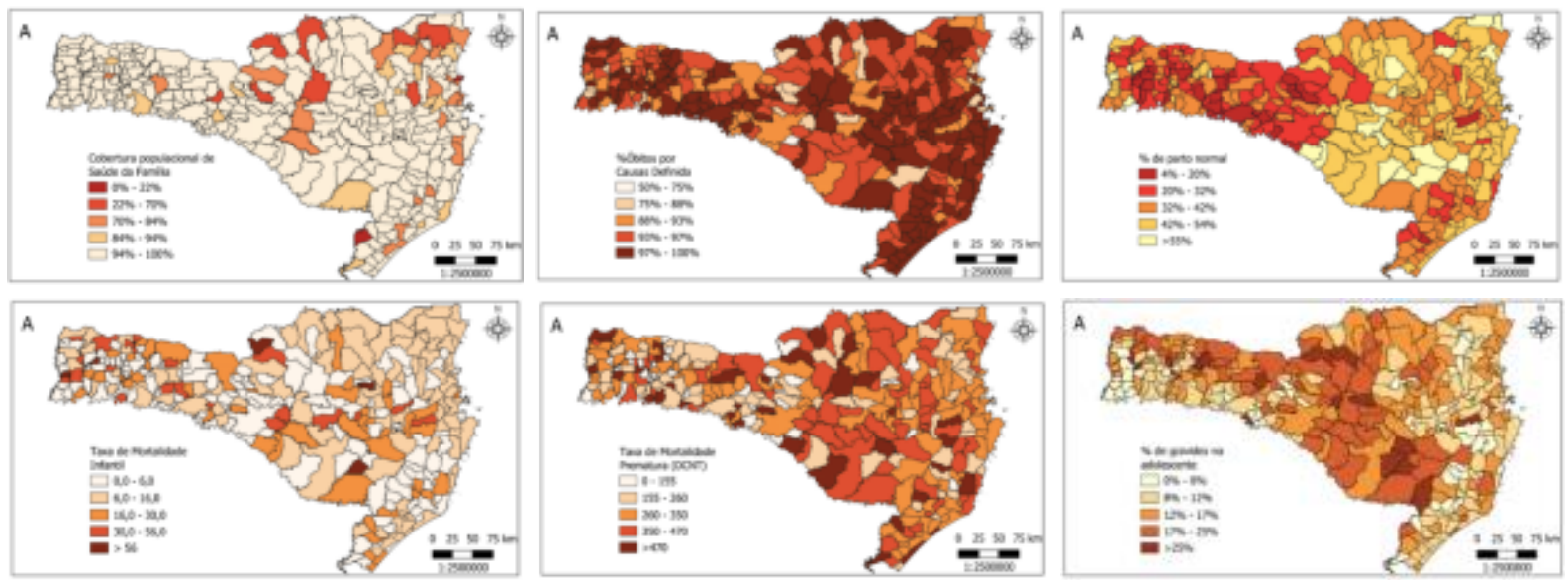

2018
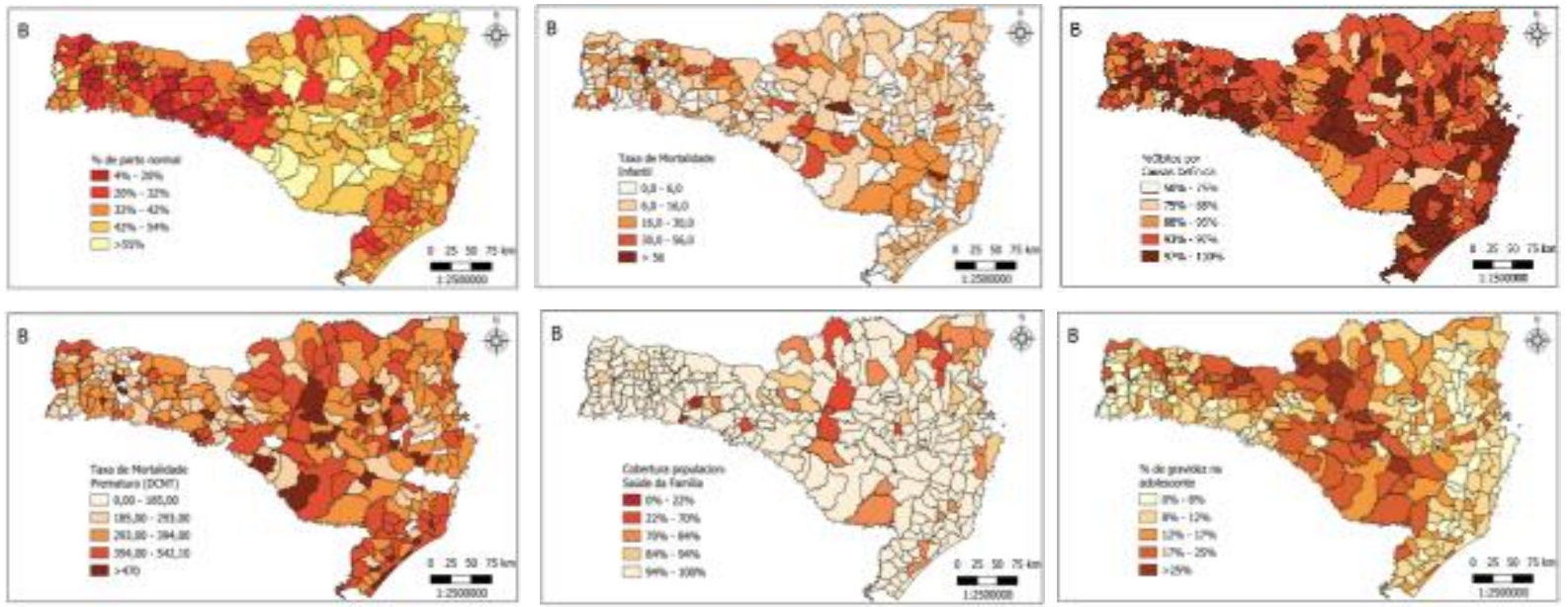

Fonte: Pesquisadores, 2020. DISCUSSÃO 
Os resultados do estudo apontam que em Santa Catarina, a maioria dos indicadores da pactuação interfederativa apresentou médias iguais ou melhores em 2018 quando comparadas a 2017. A literatura não apresenta estudos que mostrem a série temporal dos indicadores da pactuação interfederativa, 0 que dificulta comparações desses achados com outros entes federativos. No entanto, pode-se inferir que, sendo estes indicadores elementos importantes para avaliação da assistência à saúde, o estado tem buscado alcançar as metas estabelecidas, principalmente nos indicadores relacionados à promoção da saúde, redução da mortalidade infantil, assistência à gestante, responsabilidades gerais como as ações de matriciamento e à saúde do trabalhador.

Apesar das metas terem sido definidas considerando os parâmetros da literatura nacional e internacional ${ }^{2}$, os resultados dos indicadores alcançados mostram ainda, que há uma lacuna no cumprimento dessas metas e que elas precisam ser profundamente trabalhadas no planejamento do SUS. Uma vez que esses indicadores, não são simplesmente nurmeros, mas atribuiçoros de valor a objetivos, acontecimentos ou situações, de acordo com o resultado final pretendido $^{2}$.

Os resultados do estudo apontam que nesse Estado apesar da cobertura populacional estimada pelas equipes de atenção básica não ter influenciado em todos os indicadores interfederativos, ela se correlacionou significativamente com muitos deles: cobertura populacional de saúde da família (V21), percentual de vacina com cobertura adequada (V8), razão de exames citopatológicos de mulheres na faixa etária de 29-59 anos (V14), razão de exames de mamografia em mulheres na faixa etária de 49-69 anos (V15) e cobertura do programa bolsa família (V22) estão diretamente correlacionados à Cobertura populacional estimada pelas equipes de Atenção Básica (V23), tanto para o ano de 2017 quanto para o ano de 2018.

Embora a literatura também não apresente estudos que meçam diretamente o impacto da cobertura populacional pelas equipes de atenção básica em outros indicadores de saúde, há evidências da importância desse indicador no contexto da APS ${ }^{11-13}$. Nesse sentido, a literatura também traz evidências de que uma melhor cobertura populacional aprimora a política de 
atenção básica, especializada, ambulatorial e hospitalar no âmbito do SUS. ${ }^{14}$

Tal proposição se valida quando se observa que o número de óbitos por doença crônica não transmissível está inversamente correlacionado à cobertura populacional, potencializando estudos que já destacam a atuação adequada da atenção primária na redução de óbitos por doenças crônicas não transmissíveis ${ }^{9}$, pelo perfil de ações com enfoque educativo e que estão intimamente ligadas ao melhor autocuidado e manejo das comorbidades pelos usuários ${ }^{11-13}$.

Nessa vertente, os casos novos de sífilis e aids em menores de um ano de idade não perdem destaque, pois embora se refiram às infecções de caráter transmissível, também estão inversamente correlacionados à cobertura populacional, destacando o papel da atenção primária e a vigilância em saúde no monitoramento e controle da cadeia de transmissão de doenças ${ }^{12}$.

Mais do que propor a compreensão da relação da cobertura populacional estimada pelas equipes de atenção básica a alguns indicadores interfederativos, busca-se neste estudo relativizar a política de atenção primária a qual, por vezes, deixa a cargo do gestor a tomada de decisão sobre questões necessárias e que interferem diretamente no aumento da cobertura populacional, como a contratação de recursos humanos e completude das equipes que possam levar a outros desfechos na cobertura e por conseguinte, na inversão destas variáveis $^{12,15}$.

Há evidências de que uma melhor cobertura populacional pelas equipes de Atenção Básica ordena o cuidado no sistema de saúde e favorece sua capacidade resolutiva ${ }^{14-16}$.

Mesmo diante deste cenário positivo, há que se ressaltar que houve nos anos de 2017 e 2018, indicadores que não atingiram as metas. A mortalidade prematura por DCNT, por exemplo, mesmo tendo correlação inversa com a cobertura foi um dos indicadores que não atingiram as metas propostas (meta: 283 em 2017 e 277 em 2018), tendo o Estado como um todo, alcançado em 2017 283,60 e em 2018 $293,10^{17}$. Dentre os indicadores diretamente correlacionados, a cobertura vacinal também ficou aquém das metas (meta: 94,3\% em 2017 e maior que $75 \%$ em 2018), tendo o Estado como um todo alcançado em 2017 e em 2018 "0" (zero) ${ }^{17}$. Este valor possivelmente 
está relacionado aos problemas identificados nacionalmente quanto ao registro no sistema de informação de imunização (SIPNI) ${ }^{18}$,

Ainda que haja correlação favorável de indicadores interfederativos com a cobertura populacional da atenção básica nesse Estado como um todo, os mapas temáticos evidenciam que nem sempre os municípios com melhor cobertura populacional da atenção básica apresentam os melhores indicadores. Estudo anterior ${ }^{2}$ que confirmou a hipótese de que a cobertura universal é um elemento chave para alcançar objetivos globais, também evidenciou piores indicadores de saúde (relacionados à TB) em municípios com maior cobertura da Estratégia Saúde da Família (ESF).

Essas dificuldades no alcance dos indicadores são uma realidade e apontadas já em outros estudos, demonstrando a necessidade de ações no sentido de fortalecer o sistema de saúde ${ }^{2,19,20}$. Resultados encontrados neste estudo fortalecem a importância de que o planejamento dos processos de trabalho dos profissionais de saúde pode refletir, diretamente, na população atendida. Este estudo corrobora com a recomendação para o fortalecimento das diretrizes institucionais nesse Estado, em consonância com as diretrizes do SUS, com garantia de cobertura populacional, pelas equipes de Atenção Básica ${ }^{19}$.

Dentre as limitações deste estudo, destacam-se possíveis eventos não identificados, pelo perfil de dados utilizados, dados secundários. Essa limitação não reduz a confiabilidade do estudo, mas deve ser considerada na interpretação dos achados.

\section{CONSIDERAÇÕES FINAIS}

O estudo apresentou três blocos de observação, o primeiro ocupou-se em analisar os indicadores diretamente correlacionados à cobertura de Atenção Básica, o segundo, ocupou-se em analisar os indicadores inversamente correlacionados à cobertura de Atenção Básica e o terceiro, os que não foram influenciados pela cobertura.

Apesar da cobertura populacional estimada pelas equipes de atenção básica não influenciaram todos os indicadores interfederativos, vê-se que há uma relação positiva se comparado à melhoria de alguns deles, como o de cobertura de vacinação, exame citopatológico, razão de mamografia e cobertura do programa 
bolsa família, assim como, se considerarmos que os óbitos por doenças crônicas não transmissíveis, os casos novos de sífilis em menores de um ano de idade e os casos novos de aids em menores de um ano apresentam-se inversamente correlacionados à cobertura da Atenção Básica, e também valida positivamente à hipótese de que, quanto maior a cobertura de atenção básica melhor é o desempenho dos indicadores de saúde.

Outrossim, pode-se observar que o desempenho dos municípios com melhor cobertura de Atenção Básica nem sempre apresentam os melhores indicadores de saúde, o que demonstra fragilidades na organização das ações e serviços de saúde na gestão estadual e municipal. Nesse sentido, espera-se que este estudo proponha um olhar minucioso acerca da tomada de decisão dos gestores quanto a Cobertura populacional estimada pelas equipes de Atenção Básica e a necessidade de recursos humanos compatíveis aos indicadores que de fato devam ter atenção na APS bem como da organização dos processos de trabalho.

\section{REFERÊNCIA}

1. Mazzi RAP, Marques HR. Cuidados paliativos oncológicos domiciliares como uma nova prática em saúde influenciando no desenvolvimento local. Interações. 2018; 19(4):72738.

2. Souza SS. Acesso e cobertura na Atenção Primária à Saúde: uma análise dos indicadores de tuberculose no Brasil. Enfermería Comunitaria. 2019; 15:e12395.

3. Recktenwaldt $M$, Junges JR. A organização e a prática da Vigilância em Saúde em municípios de pequeno porte. Saúde Soc. 2017; 26(2):36781.

4. Lima KWS, Antunes JLF, Da Silva ZP. Percepção dos gestores sobre o uso de indicadores nos serviços de saúde. Saúde Soc. 2015; 24(1):61-71.

5. Ministério da Saúde (BR). Manual de planejamento no SUS /Ministério da Saúde, Fundação Oswaldo Cruz. Brasília (DF): Ministério da Saúde; 2016.

6. Comissão Intergestores Tripartite. Resolução CIT No 08/2016. Pactuação interfederativa 2017/2021. Indicadores para pactuação de metas.

7. Carrapato P, Correia P, Garcia B. Determinante da saúde no Brasil: a 
procura da equidade na saúde. Saúde Soc. 2017; 26(3):676-89.

8. De Carvalho ALB, Souza MF, Shimizu HE, Senra IMVB, Oliveira KC. A gestão do SUS e as práticas de monitoramento e avaliação: possibilidades e desafios para a construção de uma agenda estratégica. Ciênc Saúde Colet. 2012; 17(4):901-11.

9. Diretoria de Vigilância Epidemiológica (SC). Secretaria de Saúde de Santa Catarina. Tabnet [Internet]. 2019 [acesso em 2021 fev 05]. Disponível em: http://tabnet.dive.sc.gov.br/.

10. QGIS Development Team. QGIS Geographic Information System. Open Source Geospatial Foundation Project [Internet] 2015 [acesso em 2019 dez 01]. Disponível em: http://www.qgis.org/.

11. Guimarães RM. A teoria da equidade reversa se aplica na atenção primária à saúde? Evidências de 5564 municípios brasileiros. Rev panam salud pública. 2018; 24; 42: e128.

12. Borges DB, Lacerda JT. Ações voltadas ao controle do Diabetes Mellitus na Atenção Básica: proposta de modelo avaliativo. Saúde debate. 2018; 42(116):162-78.
13. Albuquerque $\mathrm{C}$, Martins $M$. Indicadores de desempenho no Sistema Único de Saúde: uma avaliação dos avanços e lacunas. Saúde debate. 2017; 41(spe):118-37.

14. Malta DC, Santos MAS, Stopa SR, Vieira JEB, Melo EA, Reis AAC. A Cobertura da Estratégia de Saúde da Família (ESF) no Brasil, segundo a Pesquisa Nacional de Saúde, 2013. Ciênc Saúde Colet. 2016; 21(2):32738.

15. Santos L. Região de saúde e suas redes de atenção: modelo organizativo-sistêmico do SUS. Ciênc Saúde Colet. 2017; 22(4):1281-9.

16. Ribeiro $\mathrm{TH}$, Magri $\mathrm{CL}$, Santos $\mathrm{AL}$. Hospitalizações por Diabetes Mellitus em adultos e relação com expansão Atenção Primária no Paraná. Saúde pesqui. 2019; 12(2):323-31.

17. Governo de Santa Catarina (SC). Secretaria do Estado de Saúde. Comissão Intergestores Bipartite. Deliberação $087 / \mathrm{ClB} / 2020$. Aprova as metas do rol dos indicadores interfederativos do Estado de Santa Catarina, período para 2020, conforme resolução CIT 08 de 24 de novembro de 2016, que dispõe sobre - processo de pactuação interfederativa indicadores para o 
período de 2017 a 2021 de acordo com as prioridades nacionais de saúde. Agosto de 2020.

18. Silva BS, Souza KC, Souza RG, Rodrigues SB, Oliveira VC, Guimarães EAA. Condições de estrutura e processo na implantação do Sistema de Informação de Imunização do Brasil. Rev bras enferm. 2020; 73(4):e20180939.

19. Suplici SER, Souza SS, Cunha AC, Zamprogna KM, Laurindo DLP. Associação entre casos de Aids em menores de cinco anos e cobertura da atenção básica. Rev enferm Cent Oeste Min. 2020; 10:e3908.

20. Souza SS, Cunha AC, Suplici SER, Zamprogna KM, Laurindo DLP. Influência da cobertura da Atenção Primária no enfrentamento da COVID-19. J Health NPEPS. 2021; 6(1):1-21.

Financiamento: Os autores declaram que não houve financiamento.

Conflito de interesses: Os autores declaram não haver conflito de interesses.

Participação dos autores:

Concepção: Souza SS, Palha PF, Cunha AC, Laurindo DLP, Suplici SER, Zamprogna KM.

Desenvolvimento: Souza SS, Palha PF, Cunha AC, Laurindo DLP, Suplici SER, Zamprogna KM.

Redação e revisão: Souza SS, Palha PF, Cunha AC, Laurindo DLP, Suplici SER, Zamprogna KM.

Como citar este artigo: Souza SS, Palha PF, Cunha AC, Laurindo DLP, Suplici SER, Zamprogna KM. Avaliação da atenção básica: estudo ecológico dos indicadores de pactuação em Santa Catarina. J Health NPEPS. 2021; 6(1):122-137. 